\title{
Stratum Group Number
}

National Cancer Institute

\section{Source}

National Cancer Institute. Stratum Group Number. NCI Thesaurus. Code C94082.

An integer that identifies the stratum group to study personnel, such as the statistician and registrars. This index is used to cross-reference the stratum group and set of arms during registration. It is provided to perform a lookup in the randomization book or statistical algorithm when performing randomization. 